\title{
A LOWER BOUND IN THE TAIL LAW OF THE ITERATED LOGARITHM FOR LACUNARY TRIGONOMETRIC SERIES
}

\author{
SANTOSH GHIMIRE AND CHARLES N. MOORE
}

(Communicated by Alexander Iosevich)

\begin{abstract}
Salem and Zygmund obtained an upper bound for a tail law of the iterated logarithm for sums of the form $\sum_{k=N}^{\infty} a_{k} \cos \left(n_{k} x\right)+b_{k} \sin \left(n_{k} x\right)$, where $n_{k}$ satisfies a Hadamard gap condition and $\sum_{k=1}^{\infty} a_{k}^{2}+b_{k}^{2}<\infty$. Here we obtain a lower bound in their result under the same hypotheses.
\end{abstract}

\section{INTRODUCTION AND MAIN RESULT}

The law of the iterated logarithm (LIL) first arose in the work of Khintchine [5] who sought to obtain the exact rate of convergence in Borel's theorem on normal numbers. This result was generalized by Kolmogorov [6] to sums of independent random variables.

Recall that an increasing sequence of positive numbers $\left\{n_{k}\right\}$ is said to satisfy the Hadamard gap condition if there exists a $q>1$ such that $\frac{n_{k+1}}{n_{k}}>q$ for all $k$. A trigonometric series which has the form $S(\theta)=\sum_{k=1}^{\infty} a_{k} \cos \left(n_{k} \theta\right)+b_{k} \sin \left(n_{k} \theta\right)$, where $n_{k}$ satisfies a Hadamard gap condition and $a_{k}, b_{k}$ are real, is often called a $q$-lacunary series. Let $S_{m}(\theta)$ denote the $m$ th partial sum of the series. Salem and Zygmund [7] obtained an LIL in the context of a lacunary trigonometric series:

Theorem 1 (Salem and Zygmund). Suppose that $S(\theta)$ is a q-lacunary series and $n_{k}$ are positive integers. Set $B_{m}^{2}=\frac{1}{2} \sum_{k=1}^{m}\left(a_{k}^{2}+b_{k}^{2}\right)$ and $M_{m}=\max _{1 \leq k \leq m}\left(a_{k}^{2}+b_{k}^{2}\right)^{\frac{1}{2}}$. Suppose also that $B_{m} \rightarrow \infty$ as $m \rightarrow \infty$ and $M_{m}$ satisfies the Kolmogorov-type condition: $M_{m}^{2} \leq K_{m} \frac{B_{m}^{2}}{\log \log \left(e^{e}+B_{m}^{2}\right)}$ for some sequence of numbers $K_{m} \downarrow 0$. Then

$$
\limsup _{m \rightarrow \infty} \frac{S_{m}(\theta)}{\sqrt{2 B_{m}^{2} \log \log B_{m}}} \leq 1
$$

for almost every $\theta$ in the unit circle $T$.

Toward a lower bound, progress was first made by Erdős and Gál [3. Later, M. Weiss [8] gave a complete analogue of Kolmogorov's LIL in this setting.

Theorem 2 (M. Weiss). Suppose $S_{m}(\theta)=\sum_{k=1}^{m}\left(a_{k} \cos n_{k} \theta+b_{k} \sin n_{k} \theta\right)$ is a qlacunary series. Set $B_{m}=\left(\frac{1}{2} \sum_{k=1}^{m}\left(a_{k}^{2}+b_{k}^{2}\right)\right)^{\frac{1}{2}}$ and $M_{m}=\max _{1 \leq k \leq m}\left(a_{k}^{2}+b_{k}^{2}\right)^{\frac{1}{2}}$. Suppose also that $B_{m} \rightarrow \infty$ as $m \rightarrow \infty$ and $M_{m}$ satisfies the Kolmogorov-type

Received by the editors May 21, 2012 and, in revised form, October 8, 2012.

2010 Mathematics Subject Classification. Primary 42A55; Secondary 60F15.

Key words and phrases. Law of the iterated logarithm, lacunary trigonometric series. 
condition: $M_{m}^{2} \leq K_{m} \frac{B_{m}^{2}}{\log \log \left(e^{e}+B_{m}^{2}\right)}$ for some sequence of numbers $K_{m} \downarrow 0$. Then

$$
\limsup _{m \rightarrow \infty} \frac{S_{m}(\theta)}{\sqrt{2 B_{m}^{2} \log \log B_{m}}}=1
$$

for almost every $\theta$ in the unit circle.

In their paper [7, Salem and Zygmund also introduced a "tail" law of the iterated logarithm for lacunary series. In this LIL, they considered the tail sums of convergent series instead of partial sums of divergent series. We state their result:

Theorem 3 (Salem and Zygmund). Let $\tilde{S}_{N}(\theta)=\sum_{k=N}^{\infty}\left(a_{k} \cos n_{k} \theta+b_{k} \sin n_{k} \theta\right)$ where $\frac{n_{k+1}}{n_{k}}>q>1$ and $c_{k}^{2}=a_{k}^{2}+b_{k}^{2}$ satisfies $\sum_{k=1}^{\infty} c_{k}^{2}<\infty$. Define $\tilde{B}_{N}=$ $\left(\frac{1}{2} \sum_{k=N}^{\infty} c_{k}^{2}\right)^{\frac{1}{2}}$ and $\tilde{M}_{N}=\max _{k \geq N}\left|c_{k}\right|$. Suppose that $\tilde{B}_{1}<\infty$ and that $\tilde{M}_{N}^{2} \leq$ $K_{N}\left(\frac{\tilde{B}_{N}^{2}}{\log \log \frac{1}{\tilde{B}_{N}}}\right)$ for some sequence of numbers $K_{N} \downarrow 0$ as $N \rightarrow \infty$. Then

$$
\limsup _{N \rightarrow \infty} \frac{\tilde{S}_{N}(\theta)}{\sqrt{2 \tilde{B}_{N}^{2} \log \log \frac{1}{\tilde{B}_{N}}}} \leq 1
$$

for a.e. $\theta \in[0,2 \pi]$.

In this paper we will obtain a lower bound in Salem and Zygmund's tail law of the iterated logarithm. Our main result is:

Theorem 4. With the same notation and hypotheses as in the previous theorem, we have

$$
\limsup _{N \rightarrow \infty} \frac{\tilde{S}_{N}(\theta)}{\sqrt{2 \tilde{B}_{N}^{2} \log \log \frac{1}{\tilde{B}_{N}}}} \geq 1
$$

for a.e. $\theta \in[0,2 \pi]$.

The proof will make use of a mix of techniques used by Salem and Zygmund combined with ideas from the study of dyadic martingales. We remark that the proof we give can also be adapted to give a proof of the lower bound in the M. Weiss LIL which is different from the original. We first fix some notation and state some lemmas which will be used in the course of the proof.

A dyadic subinterval of the unit interval $[0,1]$ is an interval of the form $\left[\frac{j}{2^{n}}, \frac{j+1}{2^{n}}\right)$, where $n=0,1,2, \ldots$ and $j=0,1, \ldots, 2^{n}-1$. As is to be expected, we will need a Borel-Cantelli-type lemma for independent, or at least weakly dependent, random variables. This is provided by the following, whose proof can be found in Bañuelos and Moore [1, p. 79]:

Lemma 5. For $k=1,2, \ldots$, suppose $F_{k}$ is a collection of dyadic cubes whose union is $[0,1]$ such that $F_{k+1}$ is a refinement of $F_{k}$. Suppose that the maximum length of the elements of $F_{k}$ tends to zero. Suppose $\mathcal{E}_{k} \subset F_{k}$ has the property

$$
\forall Q \in F_{k}, \quad\left|Q \cap \bigcup_{J \in \mathcal{E}_{k+1}} J\right|>|Q| \frac{C}{k} .
$$

Set $E_{k}=\bigcup_{J \in \mathcal{E}_{k}}$ J. Then for a.e. $x, x \in E_{k}$ i.o. 
It is well known that lacunary series exhibit central limit theorem-type behavior. The following, due to Gaposhkin [4, will give us this type of behavior for trigonometric polynomials with a favorable error.

Theorem 6 (Gaposhkin). Suppose that $n_{k}$ satisfies $\frac{n_{k+1}}{n_{k}} \geq q>1$ and $\lambda_{N k}$ satisfies the following conditions:

$$
\sum_{k=1}^{\infty} \lambda_{N k}^{2}=1, \quad \Lambda_{N}=\max _{k}\left|\lambda_{N k}\right|=o(1) \quad \text { as } \quad N \rightarrow \infty .
$$

Set $F_{N}(y)=P\left(\sum_{k=1}^{\infty} \lambda_{N k} \omega_{n_{k}}<y\right)$, where $\omega_{n_{k}}=\sqrt{2} \cos \left(2 \pi n_{k} x\right)$. Then the distributions $F_{N}(y)$ converge towards the normal law; moreover,

$$
\sup _{-\infty<y<\infty}\left|F_{N}(y)-\Phi(y)\right| \leq c(q) \Lambda_{N}^{1 / 4},
$$

where $\Phi(y)=\frac{1}{\sqrt{2 \pi}} \int_{-\infty}^{y} e^{-\frac{t^{2}}{2}} d t$ and the constant $c(q)$ depends only on $q$.

Finally, we need a classical result on the exponential square integrability of lacunary series (see Zygmund [9], vol. I, p. 215).

Theorem 7. Consider the series $g(x)=\sum_{k=1}^{\infty}\left(a_{k} \cos n_{k} x+b_{k} \sin n_{k} x\right)$ with $\alpha^{2}=$ $\sum_{k=1}^{\infty}\left(a_{k}^{2}+b_{k}^{2}\right)$, where $\frac{n_{k+1}}{n_{k}} \geq q>1$. If $\alpha \leq 1$, then $\int_{0}^{2 \pi} \exp \left(C g^{2}\right) d x \leq A$, provided $C \leq C_{0}(q)$, with $A$ an absolute constant.

\section{THE PROOF OF THE THEOREM}

Proof. We note that we may write

$$
a_{k} \cos n_{k} x+b_{k} \sin n_{k} x=\sqrt{a_{k}^{2}+b_{k}^{2}} \cos \left(n_{k} x-\phi_{k}\right) .
$$

We consider cosine series of the form $\sum_{k=1}^{\infty} a_{k} \cos \left(n_{k} x\right)$; the proof is identical in the general case. To facilitate working with dyadic cubes, we rescale and consider $\sum_{k=1}^{\infty} a_{k} \cos \left(2 \pi n_{k} x\right)$ on $[0,1]$.

Let $M$ be a fixed large positive number. Define $N_{1} \leq N_{2} \leq \cdots$ by

$$
N_{l}=\min \left\{N: \frac{1}{2} \sum_{k=N+1}^{\infty} a_{k}^{2}<\frac{1}{M^{l}}\right\} .
$$

Let $\varepsilon>0$ and assume $\varepsilon \ll 1$. Choose $\delta>0$ so that $(1+\delta)\left(1-\varepsilon^{2}\right)>1$. Finally choose $0<\mu<1$ so that

$$
\frac{\mu \log (8 \sqrt{\pi})}{1+\varepsilon}-\frac{1}{2} \frac{\mu \log \mu}{1+\varepsilon}+\frac{1}{1+\varepsilon}<1 .
$$

Using the definition of $N_{l}$ and the fact that $\left|a_{N}\right|<\varepsilon \sqrt{\sum_{k=N}^{\infty} a_{k}^{2}}$, for $N$ sufficiently large, we can assume that $l$ is sufficiently large so that

$$
\left(1-\varepsilon^{2}\right) \frac{1}{M^{l}}<\frac{1}{2} \sum_{k=N_{l}+1}^{\infty} a_{k}^{2}<\frac{1}{M^{l}} .
$$

Consequently,

$$
\left(1-\varepsilon^{2}\right) M<\frac{\sum_{k=N_{l}+1}^{\infty} a_{k}^{2}}{\sum_{k=N_{l+1}+1}^{\infty} a_{k}^{2}}<\frac{M}{1-\varepsilon^{2}} .
$$


By Theorem 7 (rescaled to $[0,1]$ ) and Chebyshev's inequality we obtain

$$
\left|\left\{x \in[0,1]:\left|\sum_{k=N_{l+1}+1}^{\infty} a_{k} \cos \left(2 \pi n_{k} x\right)\right| \geq \lambda \sqrt{\sum_{k=N_{l+1}+1}^{\infty} a_{k}^{2}}\right\}\right| \leq A e^{-C \lambda^{2}},
$$

for every $\lambda>0$.

Put

$$
\lambda=\left(\sum_{k=N_{l+1}+1}^{\infty} a_{k}^{2}\right)^{-\frac{1}{2}} \sqrt{\frac{(1+\delta)}{C M} \sum_{k=N_{l}+1}^{\infty} a_{k}^{2} \log \log \frac{1}{\sqrt{\frac{1}{2} \sum_{k=N_{l}+1}^{\infty} a_{k}^{2}}}}
$$

and use (2.2) to obtain the estimate

$$
\begin{aligned}
\mid\{x & \in[0,1]:\left|\sum_{k=N_{l+1}+1}^{\infty} a_{k} \cos \left(2 \pi n_{k} x\right)\right| \\
& \left.>\sqrt{\frac{(1+\delta)}{C M} \sum_{k=N_{l}+1}^{\infty} a_{k}^{2} \log \log \frac{1}{\sqrt{\frac{1}{2} \sum_{k=N_{l}+1}^{\infty} a_{k}^{2}}}}\right\} \mid \\
& \leq A \exp \left(-(1+\delta)\left(1-\varepsilon^{2}\right) \log \log M^{\frac{l}{2}}\right)=A\left(l \frac{\log M}{2}\right)^{-(1+\delta)\left(1-\varepsilon^{2}\right)} .
\end{aligned}
$$

Since $(1+\delta)\left(1-\varepsilon^{2}\right)>1$, by the Borel-Cantelli lemma for a.e. $x$ we have

$$
\frac{\left|\sum_{k=N_{l+1}+1}^{\infty} a_{k} \cos \left(2 \pi n_{k} x\right)\right|}{\sqrt{\sum_{k=N_{l}+1}^{\infty} a_{k}^{2} \log \log \frac{1}{\sqrt{\frac{1}{2} \sum_{k=N_{l}+1}^{\infty} a_{k}^{2}}}}} \leq \sqrt{\frac{1+\delta}{C M}}
$$

for all sufficiently large $l$ (depending on $x$ ). The definition of $N_{l}$ and (2.1) yields

$$
\frac{1}{2} \sum_{k=N_{l}+1}^{N_{l+1}} a_{k}^{2}>\frac{1}{M^{l}}\left(1-\varepsilon^{2}-\frac{1}{M}\right)>\left(\frac{1}{2} \sum_{k=N_{l}+1}^{\infty} a_{k}^{2}\right)\left(1-\varepsilon^{2}-\frac{1}{M}\right) .
$$

By hypotheses, for all sufficiently large $N$,

$$
\max _{k \geq N} a_{k}^{2} \leq K_{N}\left(\frac{\frac{1}{2} \sum_{k=N}^{\infty} a_{k}^{2}}{\log \log \frac{1}{\sqrt{\frac{1}{2} \sum_{k=N}^{\infty} a_{k}^{2}}}}\right) \leq \frac{\varepsilon}{2}\left(\frac{\frac{1}{2} \sum_{k=N}^{\infty} a_{k}^{2}}{\log \log \frac{1}{\sqrt{\frac{1}{2} \sum_{k=N}^{\infty} a_{k}^{2}}}}\right) .
$$

Then for $l$ for large enough,

$$
\max _{k \geq N_{l}+1} a_{k}^{2}<\frac{K_{N_{l}+1}}{\left(1-\varepsilon^{2}-\frac{1}{M}\right)} \frac{1}{\log l} \frac{1}{2} \sum_{k=N_{l}+1}^{N_{l+1}} a_{k}^{2}<\frac{\varepsilon / 2}{\left(1-\varepsilon^{2}-\frac{1}{M}\right)} \frac{1}{\log l} \frac{1}{2} \sum_{k=N_{l}+1}^{N_{l+1}} a_{k}^{2} .
$$

We may assume that $\varepsilon$ is small enough and $M$ large enough so that $1-\varepsilon^{2}-\frac{1}{M}>\frac{1}{2}$. Thus,

$$
\max _{k \geq N_{l}+1} \frac{\left|a_{k}\right|}{\sqrt{\frac{1}{2} \sum_{k=N_{l}+1}^{N_{l+1} a_{k}^{2}}}} \leq \sqrt{\frac{2 K_{N_{l}+1}}{\log l}} \leq \sqrt{\frac{\varepsilon}{\log l}}
$$


Suppose $l$ is large so that $\mu \log l \gg 1$. We define a sequence of positive integers $l_{1}, l_{2}, \cdots, l_{[]}$, where for simplicity we write []$=\left[\frac{\mu \log l}{1+\varepsilon}\right]$ ([] represents the greatest integer function) as follows:

Let $l_{1}$ be the first time such that

$$
\frac{1}{2} \sum_{k=N_{l}+1}^{N_{l}+l_{1}} a_{k}^{2} \geq \frac{1}{\mu \log l} \frac{1}{2} \sum_{k=N_{l}+1}^{N_{l+1}} a_{k}^{2}
$$

so that

$$
\frac{1}{2} \sum_{k=N_{l}+1}^{N_{l}+l_{1}-1} a_{k}^{2}<\frac{1}{\mu \log l} \frac{1}{2} \sum_{k=N_{l}+1}^{N_{l+1}} a_{k}^{2}
$$

Likewise, let $l_{2}$ be the first time such that

$$
\frac{1}{2} \sum_{k=N_{l}+l_{1}+1}^{N_{l}+l_{2}} a_{k}^{2} \geq \frac{1}{\mu \log l} \frac{1}{2} \sum_{k=N_{l}+1}^{N_{l+1}} a_{k}^{2}
$$

so that

$$
\frac{1}{2} \sum_{k=N_{l}+l_{1}+1}^{N_{l}+l_{2}-1} a_{k}^{2}<\frac{1}{\mu \log l} \frac{1}{2} \sum_{k=N_{l}+1}^{N_{l+1}} a_{k}^{2} .
$$

Similarly we define $l_{3}, \ldots, l_{[]}$. By (2.5) we have $\forall j=1,2, \cdots$, [] with $l_{0}=0$,

$$
\frac{1}{\mu \log l} \frac{1}{2} \sum_{k=N_{l}+1}^{N_{l+1}} a_{k}^{2} \leq \frac{1}{2} \sum_{k=N_{l}+l_{j-1}+1}^{N_{l}+l_{j}} a_{k}^{2}<(1+\varepsilon) \frac{1}{\mu \log l} \frac{1}{2} \sum_{k=N_{l}+1}^{N_{l+1}} a_{k}^{2} .
$$

Adding these from $j=1$ to $j=[]$ gives

$$
\frac{1}{2} \sum_{k=N_{l}+1}^{N_{l}+l_{[]}} a_{k}^{2} \leq\left[\frac{\mu \log l}{1+\varepsilon}\right] \frac{1+\varepsilon}{\mu \log l} \frac{1}{2} \sum_{k=N_{l}+1}^{N_{l+1}} a_{k}^{2} \leq \frac{1}{2} \sum_{k=N_{l}+1}^{N_{l+1}} a_{k}^{2},
$$

which shows that $N_{l}+l_{[1}$ does not exceed $N_{l+1}$. Consider a dyadic cube $Q$ such that $|Q|=2^{-L}$, where $L$ is chosen so that $2^{L} \leq n_{N_{l}}<2^{L+1}$. By Theorem 6 and (2.5) we have

$$
\begin{aligned}
& \frac{1}{|Q|}\left|\left\{x \in Q: \sum_{k=N_{l}+1}^{N_{l}+l_{1}} \frac{a_{k}}{\sqrt{\frac{1}{2} \sum_{k=N_{l}+1}^{N_{l}+l_{1}} a_{k}^{2}}} \cos \left(2 \pi n_{k} x\right)>\eta\right\}\right| \\
& \quad \geq \frac{1}{\sqrt{2 \pi}} \int_{\eta}^{\infty} e^{\frac{-t^{2}}{2}} d t-c(q)\left(2 \mu K_{N_{l}+1}\right)^{\frac{1}{8}} .
\end{aligned}
$$

Set $\eta=\sqrt{\frac{2}{\mu}}$. We can assume that $l$ is large enough (depending only on $q$, $\varepsilon$, and $\mu$ ) so that

$$
\frac{1}{|Q|}\left|\left\{x \in Q: \sum_{k=N_{l}+1}^{N_{l}+l_{1}} \frac{a_{k}}{\sqrt{\sum_{k=N_{l}+1}^{N_{l}+l_{1}} a_{k}^{2}}} \sqrt{2} \cos \left(2 \pi n_{k} x\right)>\sqrt{\frac{2}{\mu}}\right\}\right| \geq \frac{1}{2} \frac{1}{\sqrt{2 \pi}} \int_{\sqrt{\frac{2}{\mu}}}^{\infty} e^{\frac{-t^{2}}{2}} d t .
$$


Rearrange and use the estimate $\int_{\xi}^{\infty} e^{\frac{-u^{2}}{2}} d u \geq \frac{\xi}{1+\xi^{2}} e^{\frac{-\xi^{2}}{2}}$ (valid for $\xi>0$; see e.g. Chung [2, p. 231]) and the fact that $\mu<1$ to obtain

$$
\left|\left\{x \in Q: \sum_{k=N_{l}+1}^{N_{l}+l_{1}} a_{k} \cos \left(2 \pi n_{k} x\right)>\sqrt{\frac{2}{\mu}} \sqrt{\frac{1}{2} \sum_{k=N_{l}+1}^{N_{l}+l_{1}} a_{k}^{2}}\right\}\right| \geq \frac{1}{8 \sqrt{\pi}} \sqrt{\mu} e^{-\frac{1}{\mu}}|Q| .
$$

Let

$$
G(Q)=\left\{x \in Q: \sum_{k=N_{l}+1}^{N_{l}+l_{1}} a_{k} \cos \left(2 \pi n_{k} x\right)>\sqrt{\frac{2}{\mu}} \sqrt{\frac{1}{2} \sum_{k=N_{l}+1}^{N_{l}+l_{1}} a_{k}^{2}}\right\} .
$$

Put $h(x)=\sum_{k=N_{l}+1}^{N_{l}+l_{1}} a_{k} \cos \left(2 \pi n_{k} x\right)$. Then $\left|h^{\prime}(x)\right| \leq 2 \pi \sqrt{\sum_{k=N_{l}+1}^{N_{l}+l_{1}} a_{k}^{2}} \sqrt{\sum_{k=N_{l}+1}^{N_{l}+l_{1}} n_{k}^{2}}$.

Choose $L_{1}$ so that $2^{L_{1}} \leq n_{N_{l}+l_{1}}<2^{L_{1}+1}$. Then $\sqrt{\sum_{k=N_{l}+1}^{N_{l}+l_{1}} n_{k}^{2}} \leq 2^{L_{1}+1} \frac{q}{\sqrt{q^{2}-1}}$.

Thus,

$$
\left|h^{\prime}(x)\right| \leq 2 \pi \sqrt{\sum_{k=N_{l}+1}^{N_{l}+l_{1}}} a_{k}^{2} \sqrt{\sum_{k=N_{l}+1}^{N_{l}+l_{1}} n_{k}^{2}} \leq 2 \pi 2^{L_{1}+1} \frac{q}{\sqrt{q^{2}-1}} \sqrt{\sum_{k=N_{l}+1}^{N_{l}+l_{1}} a_{k}^{2}} .
$$

Let $x \in G(Q)$ and consider $y$ with $|x-y|<2^{-L_{1}}$. Then

$$
|h(y)| \geq|h(x)|-B \sqrt{\sum_{k=N_{l}+1}^{N_{l}+l_{1}} a_{k}^{2}}
$$

(where $B=B(q)$ ) and hence

$$
|h(y)| \geq \sqrt{\frac{2}{\mu}} \sqrt{\frac{1}{2} \sum_{k=N_{l}+1}^{N_{l}+l_{1}} a_{k}^{2}}-B \sqrt{\sum_{k=N_{l}+1}^{N_{l}+l_{1}} a_{k}^{2}}=\left(\frac{\sqrt{2}-\sqrt{2 \mu} B}{\sqrt{\mu}}\right) \sqrt{\frac{1}{2} \sum_{k=N_{l}+1}^{N_{l}+l_{1}} a_{k}^{2}} .
$$

From (2.6), this last estimate, and the definition of $l_{1}$, we conclude that there exists a collection of dyadic subcubes $\left\{Q^{\prime}\right\}$ of $Q$ with each $\left|Q^{\prime}\right|=2^{-L_{1}}$ such that $\forall x \in Q^{\prime}$

$$
\sum_{k=N_{l}+1}^{N_{l}+l_{1}} a_{k} \cos \left(2 \pi n_{k} x\right) \geq\left(\frac{\sqrt{2}-\sqrt{2 \mu} B}{\sqrt{\mu}}\right) \sqrt{\frac{1}{\mu \log l} \frac{1}{2} \sum_{k=N_{l}+1}^{N_{l+1}} a_{k}^{2}}
$$

and with $\left|\bigcup_{Q^{\prime} \subset Q} Q^{\prime}\right|>\frac{1}{8 \sqrt{\pi}} \sqrt{\mu} e^{-\frac{1}{\mu}}|Q|$.

Again on each $Q^{\prime}$ we use Theorem 6 to obtain

$$
\left|\left\{x \in Q^{\prime}: \sum_{k=N_{l}+l_{1}+1}^{N_{l}+l_{2}} a_{k} \cos \left(2 \pi n_{k} x\right)>\sqrt{\frac{2}{\mu}} \sqrt{\frac{1}{2} \sum_{k=N_{l}+l_{1}+1}^{N_{l}+l_{2}} a_{k}^{2}}\right\}\right| \geq \frac{\sqrt{\mu} e^{-\frac{1}{\mu}}}{8 \sqrt{\pi}}\left|Q^{\prime}\right| .
$$


Then as above there exists a collection of dyadic subcubes $\left\{Q^{\prime \prime}\right\}$ of $Q^{\prime}$ with $\left|Q^{\prime \prime}\right|=2^{-L_{2}}$, where $L_{2}$ satisfies $2^{L_{2}} \leq n_{N_{l}+l_{2}}<2^{L_{2}+1}$, such that $\forall x \in Q^{\prime \prime}$,

$$
\sum_{k=N_{l}+l_{1}+1}^{N_{l}+l_{2}} a_{k} \cos \left(2 \pi n_{k} x\right) \geq\left(\frac{\sqrt{2}-\sqrt{2 \mu} B}{\sqrt{\mu}}\right) \sqrt{\frac{1}{\mu \log l} \frac{1}{2} \sum_{k=N_{l}+1}^{N_{l+1}} a_{k}^{2}}
$$

and with $\left|\bigcup_{Q^{\prime \prime} \subset Q^{\prime}} Q^{\prime \prime}\right|>\frac{1}{8 \sqrt{\pi}} \sqrt{\mu} e^{-\frac{1}{\mu}}\left|Q^{\prime}\right|$. We continue this process. Eventually we come to a subcollection of cubes $\{I\}$ with $|I|=2^{-L_{[]}}$, where [] is the greatest integer contained in $\frac{\mu \log l}{1+\varepsilon}$ and $L_{[]}$is the number satisfying $2^{L_{[]}} \leq n_{N_{l}+l_{[]}}<2^{L_{[]}+1}$, such that $\forall x \in I$,

$$
\sum_{k=N_{l}+l_{[]-1}+1}^{N_{l}+l_{[]}} a_{k} \cos \left(2 \pi n_{k} x\right) \geq\left(\frac{\sqrt{2}-\sqrt{2 \mu} B}{\sqrt{\mu}}\right) \sqrt{\frac{1}{\mu \log l} \frac{1}{2} \sum_{k=N_{l}+1}^{N_{l+1}} a_{k}^{2}} .
$$

Moreover, $\left|\bigcup_{I \subset \tilde{Q}} I\right|>\frac{1}{8 \sqrt{\pi}} \sqrt{\mu} e^{-\frac{1}{\mu}}|\tilde{Q}|$, where $\tilde{Q}$ is the previous generation cube. On each such $I$, we use Theorem 8.25, Chapter 5, of [9] (rescaled to $I$ ) to conclude that

$$
\frac{1}{|I|} \mid\left\{x \in I: \sum_{k=N_{l}+l_{[]}+1}^{N_{l+1}} a_{k} \cos \left(2 \pi n_{k} x\right)>\lambda_{q} \sqrt{\frac{1}{2} \sum_{k=N_{l}+l_{[]}+1}^{N_{l+1}} a_{k}^{2}}\right\} \geq \mu_{q},
$$

where $\lambda_{q}$ and $\mu_{q}$ are positive constants depending only on $q$. Let $G(I)$ denote the above set. Choose $\tilde{L}$ so that $2^{\tilde{L}} \leq n_{N_{l+1}}<2^{\tilde{L}+1}$. Let

$$
h(x)=\sum_{k=N_{l}+l_{[]}+1}^{N_{l+1}} a_{k} \cos \left(2 \pi n_{k} x\right) .
$$

Using (2.5) we can then estimate

$$
\left|h^{\prime}(x)\right| \leq 2 \pi \sum_{k=N_{l}+l_{[]}+1}^{N_{l+1}}\left|a_{k}\right| n_{k} \leq 2 \pi \sqrt{\frac{2 K_{N_{l}+1}}{\log l}} \sqrt{\frac{1}{2} \sum_{k=N_{l}+1}^{N_{l+1}} a_{k}^{2}} \cdot \frac{2^{\tilde{L}+1} q}{q-1} .
$$

Let $x \in G(I)$ and consider $y$ such that $|x-y|<2^{-\tilde{L}}$. Then

$$
|h(y)| \geq|h(x)|-c(q) \sqrt{\frac{K_{N_{l}+1}}{\log l}} \sqrt{\frac{1}{2} \sum_{k=N_{l}+l}^{N_{l+1}} a_{k}^{2}} .
$$

Thus, there exists a collection of dyadic subcubes $\{J\}$ of $I$ with $|J|=2^{-\tilde{L}}$ such that $\forall x \in J$,

$$
\begin{aligned}
\sum_{k=N_{l}+l_{[]}+1}^{N_{l+1}} a_{k} \cos \left(2 \pi n_{k} x\right) & >\lambda_{q}-c(q) \sqrt{\frac{K_{N_{l}+1}}{\log l}} \sqrt{\frac{1}{2} \sum_{k=N_{l}+1}^{N_{l+1}} a_{k}^{2}} \\
& >-c(q) \sqrt{\frac{K_{N_{l}+1}}{\log l}} \sqrt{\frac{1}{2} \sum_{k=N_{l}+1}^{N_{l+1}} a_{k}^{2}} .
\end{aligned}
$$


Moreover, $\left|I \bigcap \bigcup_{J \subset I} J\right|>\mu_{q}|I|$.

Finally, adding the estimates from all of the above generations, we have

$$
\begin{aligned}
& \sum_{k=N_{l}+1}^{N_{l}+l_{1}} a_{k} \cos \left(2 \pi n_{k} x\right)+\cdots+\sum_{k=N_{l}+l_{[]}-1}^{N_{l}+l_{[]}} a_{k} \cos \left(2 \pi n_{k} x\right) \\
& \quad+\sum_{k=N_{l}+l_{[]}+1}^{N_{l+1}} a_{k} \cos \left(2 \pi n_{k} x\right) \\
& >\left(\left[\frac{\mu \log l}{1+\varepsilon}\right]\left(\frac{\sqrt{2}-\sqrt{2 \mu} B}{\sqrt{\mu}}\right)-c(q) \sqrt{K_{N_{l}+1} \mu}\right) \sqrt{\frac{1}{\mu \log l} \frac{1}{2} \sum_{k=N_{l}+1}^{N_{l+1}} a_{k}^{2}}
\end{aligned}
$$

on a subcollection $\{J\}$ of dyadic subcubes of $Q$ with $|J|=2^{-\tilde{L}}$ and with

$$
\begin{gathered}
|Q \cap \bigcup J|>|Q|\left(\frac{1}{8 \sqrt{\pi}} \sqrt{\mu} e^{-\frac{1}{\mu}}\right)^{\left[\frac{\mu \log l}{1+\varepsilon}\right]} \mu_{q} \geq|Q|\left(\frac{1}{8 \sqrt{\pi}} \sqrt{\mu} e^{-\frac{1}{\mu}}\right)^{\frac{\mu \log l}{1+\varepsilon}} \mu_{q} \\
=|Q| l^{-\left(\frac{\mu \log 8 \sqrt{\pi}}{1+\varepsilon}-\frac{\mu \log \mu}{2(1+\varepsilon)}+\frac{1}{1+\varepsilon}\right)} \mu_{q} \geq C \frac{|Q|}{l} .
\end{gathered}
$$

Consequently, $|Q \cap \bigcup J|>C \frac{|Q|}{l}$. We may assume that $l$ is large enough so that

$$
\left[\frac{\mu \log l}{1+\varepsilon}\right] /\left(\frac{\mu \log l}{1+\varepsilon}\right)>\frac{1}{(1+\varepsilon)^{1 / 2}}
$$

and

so that then

$$
\left(\frac{\sqrt{2}-\sqrt{2 \mu} B}{(1+\varepsilon)^{3 / 2}}\right)-c(q) \frac{\sqrt{K_{N_{l}+1}}}{\log l} \geq \frac{\sqrt{2}-\sqrt{2 \mu} B}{(1+\varepsilon)^{2}}
$$

$$
\begin{aligned}
& \left(\left[\frac{\mu \log l}{1+\varepsilon}\right]\left(\frac{\sqrt{2}-\sqrt{2 \mu} B}{\sqrt{\mu}}\right)-c(q) \sqrt{K_{N_{l}+1} \mu}\right) \sqrt{\frac{1}{\mu \log l} \frac{1}{2} \sum_{k=N_{l}+1}^{N_{l+1}} a_{k}^{2}} \\
& \geq\left(\left(\frac{\mu \log l}{(1+\varepsilon)^{3 / 2}}\right)\left(\frac{\sqrt{2}-\sqrt{2 \mu} B}{\sqrt{\mu}}\right)-c(q) \sqrt{K_{N_{l}+1} \mu}\right) \sqrt{\frac{1}{\mu \log l} \frac{1}{2} \sum_{k=N_{l}+1}^{N_{l+1}} a_{k}^{2}} \\
& =\left(\frac{\sqrt{2}-\sqrt{2 \mu} B}{(1+\varepsilon)^{3 / 2}}-c(q) \frac{\sqrt{K_{N_{l}+1}}}{\log l}\right) \sqrt{\log l \frac{1}{2} \sum_{k=N_{l}+1}^{N_{l+1}} a_{k}^{2}} \\
& \geq\left(\frac{\sqrt{2}-\sqrt{2 \mu} B}{(1+\varepsilon)^{2}}\right) \sqrt{\log l \frac{1}{2} \sum_{k=N_{l}+1}^{N_{l+1}} a_{k}^{2}} .
\end{aligned}
$$

Then

$$
\left\{x \in Q: \sum_{k=N_{l}+1}^{N_{l+1}} a_{k} \cos \left(2 \pi n_{k} x\right)>\left(\frac{\sqrt{2}-\sqrt{2 \mu} B}{(1+\varepsilon)^{2}}\right) \sqrt{\log l \frac{1}{2} \sum_{k=N_{l}+1}^{N_{l+1}} a_{k}^{2}}\right\} \supseteq \bigcup J .
$$

Thus, if we let $F_{l}$ denote the family of dyadic cubes $Q$ in $[0,1]$ of sidelength $2^{-L}$ (recall $2^{L} \leq n_{N_{l}}<2^{L+1}$ ) and let $\mathcal{E}_{l+1}$ denote the union of those cubes $J$ of sidelength $2^{-\tilde{L}}$ (recall $2^{\tilde{L}} \leq n_{N_{l+1}}<2^{\tilde{L}}$ ) found in all of $Q$ using the above argument, then, for 
large enough $l$ (depending only on $\varepsilon$ and $M$ ), the hypotheses of Lemma 5 are satisfied, so that for a.e. $x$ there exists an infinite sequence of numbers $N_{1}<N_{2}<\cdots$ such that

$$
\frac{\sum_{k=N_{l}+1}^{N_{l+1}} a_{k} \cos \left(2 \pi n_{k} x\right)}{\sqrt{\log l \frac{1}{2} \sum_{k=N_{l}+1}^{N_{l+1}} a_{k}^{2}}}>\left(\frac{\sqrt{2}-\sqrt{2 \mu} B}{(1+\varepsilon)^{2}}\right)
$$

for all $l$ sufficiently large. By (2.1), for sufficiently large $l$,

$$
\log l>\frac{\log \left(l \log \sqrt{M}-\log \sqrt{1-\varepsilon^{2}}\right)}{1+\varepsilon}>\frac{\log \log \frac{1}{\sqrt{\frac{1}{2} \sum_{k=N_{l}+1}^{\infty} a_{k}^{2}}}}{1+\varepsilon} .
$$

Then using this and (2.4) in (2.8) we have

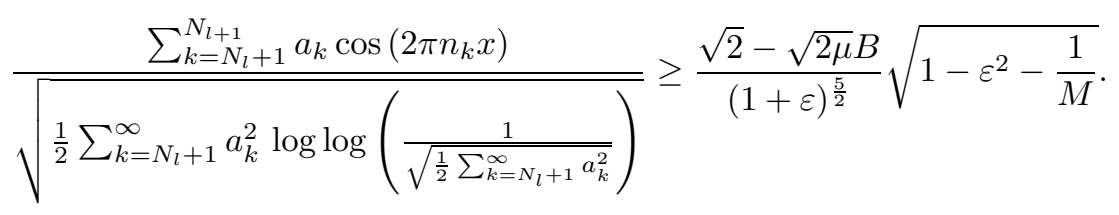

Consequently, for a.e. $x$ and large enough $l$ (depending on $x$ ),

$$
\frac{\sum_{k=N_{l}+1}^{\infty} a_{k} \cos \left(2 \pi n_{k} x\right)-\sum_{k=N_{l+1}+1}^{\infty} a_{k} \cos \left(2 \pi n_{k} x\right)}{\sqrt{\frac{1}{2} \sum_{k=N_{l}+1}^{\infty} a_{k}^{2} \log \log \left(\frac{1}{\sqrt{\frac{1}{2} \sum_{k=N_{l}+1}^{\infty} a_{k}^{2}}}\right)}} \geq \frac{\sqrt{2}-\sqrt{2 \mu} B}{(1+\varepsilon)^{5 / 2}} \sqrt{1-\varepsilon^{2}-\frac{1}{M}}
$$

But from (2.3) for a.e. $x$ we have

$$
\frac{\left|\sum_{k=N_{l+1}+1}^{\infty} a_{k} \cos \left(\pi n_{k} x\right)\right|}{\sqrt{\frac{1}{2} \sum_{k=N_{l}+1}^{\infty} a_{k}^{2} \log \log \frac{1}{\sqrt{\frac{1}{2} \sum_{k=N_{l}+1}^{\infty} a_{k}^{2}}}}} \leq \sqrt{\frac{2(1+\delta)}{C M}}
$$

for sufficiently large $l$ (depending on $x$ ).

Hence for a.e. $x$ and large enough $l$ we have

$$
\frac{\sum_{k=N_{l}+1}^{\infty} a_{k} \cos \left(2 \pi n_{k} x\right)}{\sqrt{\frac{1}{2} \sum_{k=N_{l}+1}^{\infty} a_{k}^{2} \log \log \frac{1}{\sqrt{\frac{1}{2} \sum_{k=N_{l}+1}^{\infty} a_{k}^{2}}}}} \geq \frac{\sqrt{2}-\sqrt{2 \mu} B}{(1+\varepsilon)^{5 / 2}} \sqrt{1-\varepsilon^{2}-\frac{1}{M}}-\sqrt{\frac{2(1+\delta)}{C M}} .
$$

Thus, for a.e. $x$,

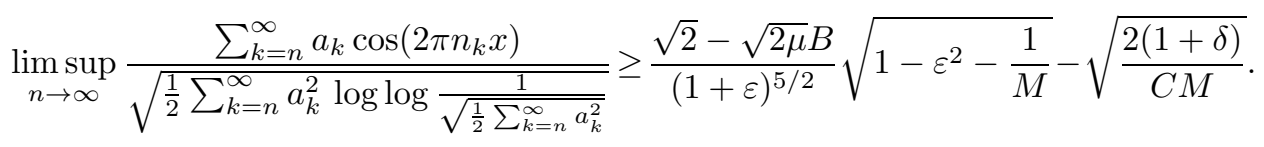

By the choice of $\delta$, we can take $\delta \searrow 0$ as $\varepsilon \searrow 0$. Also, by the choice of $\mu$, we have $\mu \searrow 0$ as $\varepsilon \searrow 0$. Then letting $M \nearrow \infty, \varepsilon \searrow 0$, and $\delta \searrow 0$ we obtain

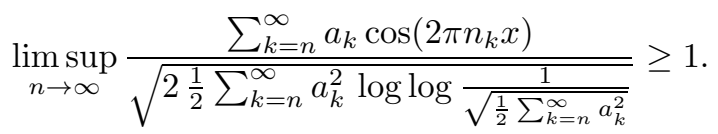




\section{REFERENCES}

[1] Rodrigo Bañuelos and Charles N. Moore, Probabilistic behavior of harmonic functions, Progress in Mathematics, vol. 175, Birkhäuser Verlag, Basel, 1999. MR1707297 (2001j:31003)

[2] Kai Lai Chung, A course in probability theory, 2nd ed., Probability and Mathematical Statistics, Vol. 21. Academic Press [A subsidiary of Harcourt Brace Jovanovich, Publishers], New York-London, 1974. MR0346858 (49 \#11579)

[3] P. Erdős and I. S. Gál, On the law of the iterated logarithm. I, II, Nederl. Akad. Wetensch. Proc. Ser. A 58 (1955), 65-84. MR0069309

[4] V. F. Gaposhkin, On the speed of convergence to the Gaussian law of weighted sums of gap series, Theory of Probability and its Applications 13 (1968), 421-438.

[5] A. Khintchine, Über einen Satz der Wahrscheinlichkeitsrechnung, Fundamenta Mathematica, 6 (1924), 9-20.

[6] N. Kolmogorov, Über des Gesetz des iterierten Logarithmus, Mathematische Annalen 101 (1929), 126-139.

[7] R. Salem and A. Zygmund, La loi du logarithme itéré pour les séries trigonométriques lacunaires (French), Bull. Sci. Math. (2) 74 (1950), 209-224. MR0039828 (12,605c)

[8] Mary Weiss, The law of the iterated logarithm for lacunary trigonometric series, Trans. Amer. Math. Soc. 91 (1959), 444-469. MR0108681 (21 \#7396)

[9] A. Zygmund, Trigonometric series. 2nd ed. Vols. I, II, Cambridge University Press, New York, 1959. MR0107776(21 \#6498)

Department of Science and Humanities, Tribhuvan University, Pulchowk Campus, LALitPuR, KATHMANDU, NePAL

E-mail address: ghimire@math.ksu.edu

Department of Mathematics, Kansas State University, Manhattan, Kansas 66506 Current address: Department of Mathematics, Washington State University, Pullman, Washington 99164

E-mail address: cnmoore@math.wsu.edu 